\title{
Téoros
}

Revue de recherche en tourisme

\section{Prévisions à court terme pour l'hôtellerie à Montréal}

\section{Paul Bodson et Jean Stafford}

Volume 8, numéro 2, juillet 1989

Hébergement et tourisme

URI : https://id.erudit.org/iderudit/1080317ar

DOI : https://doi.org/10.7202/1080317ar

Aller au sommaire du numéro

Éditeur(s)

Université du Québec à Montréal

ISSN

0712-8657 (imprimé)

1923-2705 (numérique)

Découvrir la revue

Citer cet article

Bodson, P. \& Stafford, J. (1989). Prévisions à court terme pour l'hôtellerie à Montréal. Téoros, 8(2), 3-7. https://doi.org/10.7202/1080317ar d'utilisation que vous pouvez consulter en ligne.

https://apropos.erudit.org/fr/usagers/politique-dutilisation/ 


\section{Prévisions à court terme pour I'hôtellerie à Montréal}

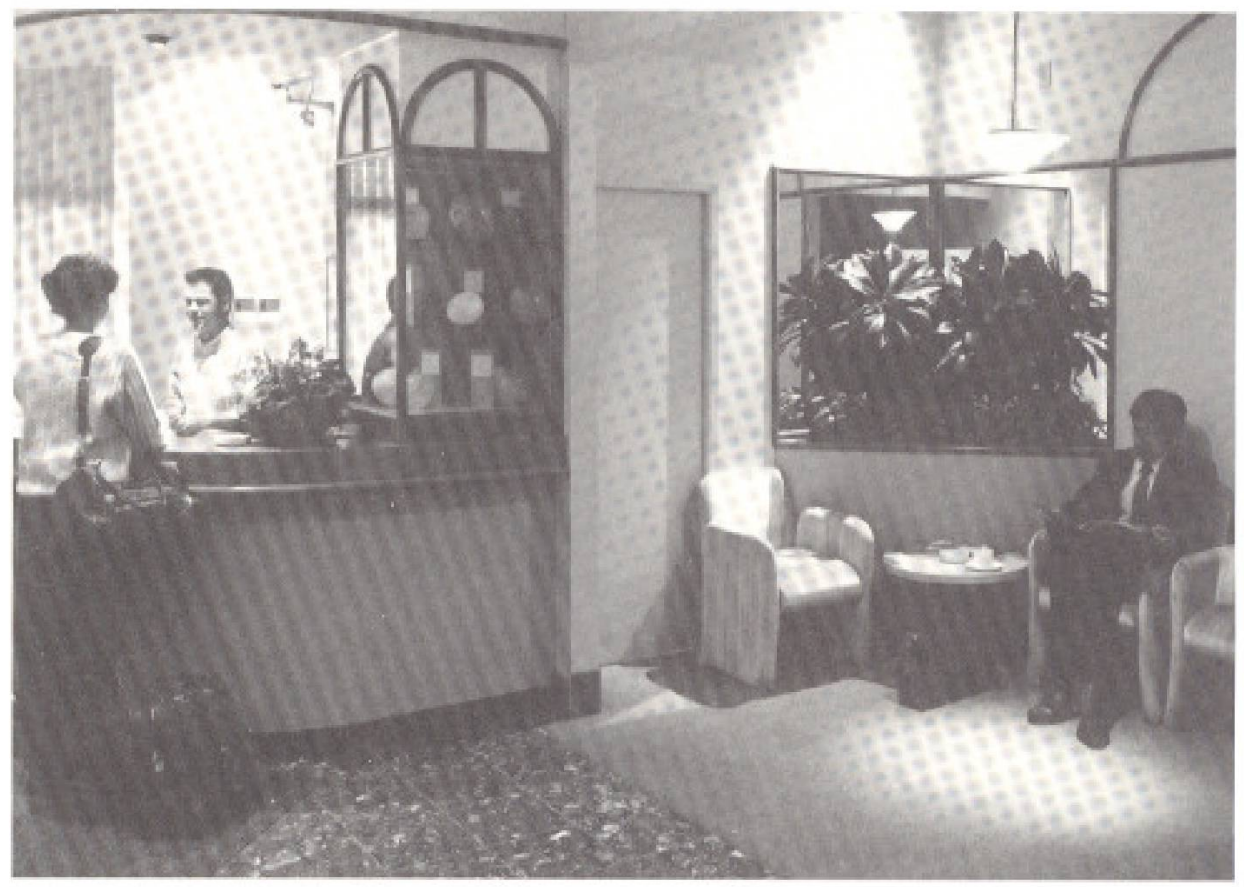

Cet article présente des previsions à court terme (pour 1989 et une partie de 1990) des chambres disponibles, des nuitéeset des taux d'occupation dans les hôtels de Montréal en les resituant dans le cadre des fluctuations inherentes à ces series chronologiques et en les référant à la structure de marché dont elles sont représentatives.

Les données exploitées pour les previsions concernent l'ensemble des hotels du Grand Montréal et selon les délimitations territoriales et les sources qu'utilise l'Association des hôtels du Grand Montréal".

Dans l'industrie hôtelière québécoise, la région de Montréal occupe une part de première importance. $61 \%$ des établissements hôteliers de 201 chambres et plus du Québec sont en effet situés dans la région touristique de Montreals.

Dans la répartition spatiale des disponibilités hồteličres de la région touristique de Moniréal, l'ìle de Montréal (Montréal-Centre inclus) détient une position majorilaire que ne semble pas entamer la pression concurrentielle des banlieues. Dans le tableau 1'a, on perçoit la relative stabilite (cn terme de proportions) des établissements et du nombre de chambres pour Montrical-Centre, l'ille de Montréal (centre exclu) et les banlieues de 1973 à 1988 . Dans Montrál-Centre, le nombre d'établissenents diminue alors que le nombre de chambres augmente; dans l'île de Montréal, les proportions restent stables alors que dans les banlieues, le nombre des élablissements augmente et le nombre de chambres diminue.

\section{Approches méthodologiques}

Pour l'industrie touristique, le besoin de prévisions rigoureuses est une préoccupation constante. Dés les années 1970 , on trouve dans la litterature scientifique, concemant le tourisme, diverses études consacrés à ce problème. Maurice Renouxi et Brian Archero s'inspirent des modeles gravitionnels pour construire des prévisions à moyen terme et à long terme. Raymond Bar On integre les mouvements suisonnierset irréguliers aux analyses prévisionnelles.

Plus recemment, certains auteurs ont fait l'inventaire des methodes prévisionnelles applicables au tourisme. II existe cn fait une batterie de techniques statistiques aptes à capter les différents mouvements des séries chronologiques et pouvant nous aider à faire des prévisions valablesw.

Danscette étude, nous avons privilégié un modele d'ajustement à un modèle explicatifi" Les sẻries temporelles peuvent se résumer par la formule suivante: $Y=f(T, C, S, I)$ où:
* Messieurs Paul Bodson et Jean Stafford sont tecourbeines et touresseurs au Departement d'etudes Montreal. 
$\mathrm{Y}=$ variable à prévoir;

$\mathrm{T}=$ tendance générale de la série:

$\mathrm{C}=$ mouvement cyclique;

$\mathrm{S}=$ mouvement saisonnier,

I = mouvement irrégulier aléatoire.

IIl s'agit bien sûr d'une approche théorique. Dans la réalité de la série temporelle, on tente de saisir certains mouvements et de déterminer leurs amplitudes afin d'effectuer les meilleures previsions possible. Nous faisons l'hypothèse implicite que ces mouvements existent et qu'ils peuvent être cermés.

Afin de bien capter les mouvements de la série ct de faire les meilleures previsions possible, nous avons utilise suecessivement 5 approches dans le traitement des données temporelles de l'hôtellerie à Montréal. La comparaison de ces diverses approches permet de déterniner le meilleur ajustement entre la série observée ef la série prevue et de faire des projections. Ces approches sont les suivantes:

1- le lissage exponentiel;

2- la méthode dite de Winters:

3- la méthode Stepar;

4- la méthode $X \| I$ associéc à la régression:

5- la méthode XI 1 associéc à la méthode Stepar.

La prévision par lisage exponentiel se fait à l'aide de moyennes mobiles pondérées; c'est une méthode empirique qui établit la prévision à partir de ponderations des informations antérieures. Le modèle de Winters est une variante du lissage exponentiel. Sa particularite est de décomposer l'erreur prévisionnelle en troiséléments que nous appelerons E1, E2 et E3:

E1 = c'est la partie de l'erreur causée par les variations de la moyenne des observations utiliscés:

E2 = c'est la partie de l'erreur induite par les variations dans la tendance;

E3 = c'est la variable aléatoire qui reste "..

La methode Stepar (Stepwise Autoregressive Methody"t est une méthode plus complexe qui associe deux processus:

un processus sous forme de tendance: $Y_{n}=\mathrm{a}+$ $b_{t}+c_{t}^{2}+c_{t}$

et un processus autorégressif: $\mathrm{Y}_{1}=\partial+\mathrm{BY}_{\mathrm{t}-1}$ + $\partial \mathrm{Y}_{\mathrm{t}-2} \ldots+\mathrm{e}_{\mathrm{t}}$

Le processus sous la forme de tendance capte le mouvement à long terme, le processus autorégressif, les fluctuations à court terme, le teme e, les fluctuations alćatoires:" Le modèle śappliquant à la série étudiếe est séllectionnê en
TABLEAU 1

Évolution en pourcentage du nombre d'établissements et du nombre de chambres pour les zones: Montréal-Centre, l'île de Montréal et les banlieues en 1973,1983 et 1988

\begin{tabular}{|c|l|c|c|c|c|}
\hline Annees & $\begin{array}{c}\text { Établissements } \\
\text { et chambres }\end{array}$ & Mil-Centre & ile de Mtl & Banlieues & Total \\
\hline $1973^{*}$ & Étab. & 34,1 & 22,3 & 43,6 & 100 \\
& Chambres & 51,2 & 25,7 & 23,1 & 100 \\
$1983^{* *}$ & Etab. & 32,5 & 25,1 & 42,4 & 100 \\
& Chambres & 58,5 & 24,2 & 17,6 & 100 \\
$1988^{*+4 *}$ & Etabl. & 30,9 & 22,1 & 47,0 & 100 \\
& Chambres & 57,4 & 23,7 & 18,9 & 100 \\
\hline
\end{tabular}

Sources:

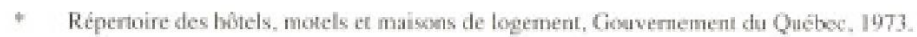

*H Hebergement Quebec, Gouvemenent du Quebec, 1987.

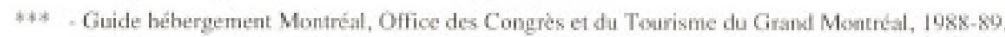

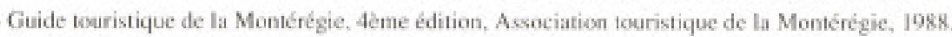

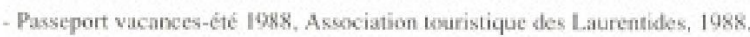

(1) La zone Montréal-Centre exclue

TABLEAU 2

Les approches prévisionnelles et les hypothèses utilisées pour I'industrie hôtelière à Montréal

\begin{tabular}{|l|c|c|c|}
\hline \multicolumn{1}{|c|}{ Hypotheses } & Stabilité lineaire & Tendance parabolique & Tendance quadratique \\
Approches & & 2 & 3 \\
\hline Lissage exponentiel & 1 & 5 & 6 \\
Winters & 4 & 8 & 9 \\
Stepar & 10 & 11 & 12 \\
Xilidécomposition) & 13 & 14 & 15 \\
XI1 et Stepar & & \\
\hline
\end{tabular}

TABLEAU 3

Évolution indicielle des chembres disponibles*, des nuitées et des taux d'occupation des hôtels de Montréal (base $100=1977$ )

\begin{tabular}{|c|c|c|c|c|c|c|}
\hline Annés & $\begin{array}{c}\text { Moyennes } \\
\text { des } \\
\end{array}$ & $\begin{array}{c}\text { Moyennes } \\
\text { des numbres } \\
\text { disponibles } \\
\text { par mois }\end{array}$ & $\begin{array}{c}\text { Moyennes } \\
\text { annuelles } \\
\text { des taux }\end{array}$ & \multicolumn{3}{|c|}{ Indices (base 100 =1977) } \\
\cline { 5 - 7 } & & & $\begin{array}{c}\text { Chambres } \\
\text { disponibles }\end{array}$ & Nuitées & Taux \\
\hline 1977 & 311532 & 189882 & 62 & 100 & 100 & 100 \\
1978 & 322242 & 204453 & 63 & 103 & 108 & 102 \\
1979 & 324344 & 220878 & 68 & 104 & 116 & 110 \\
1980 & 332641 & 227059 & 68 & 107 & 119 & 110 \\
1981 & 322260 & 215072 & 66 & 103 & 113 & 106 \\
1982 & 339864 & 202855 & 59 & 109 & 107 & 95 \\
1983 & 334728 & 201946 & 57 & 114 & 106 & 92 \\
1984 & 346268 & 218561 & 63 & 111 & 115 & 102 \\
1985 & 334080 & 231868 & 69 & 107 & 122 & 111 \\
1986 & 347841 & 239654 & 69 & 112 & 126 & 111 \\
1987 & 370745 & 256905 & 69 & 119 & 135 & 111 \\
1988 & 384427 & 264339 & 69 & 123 & 139 & 111 \\
\hline
\end{tabular}

Source: Associaion des botels du Grand Montrabl. 
TABLEAU 4

\section{Coefficients saisonniers" des chambres disponibles, des nuitées et des taux d'occupation des hôtels de Montréal (1977-1988)}

\begin{tabular}{|c|c|c|c|}
\hline Mois & Chambres disponibles & Nuitées & Taux d'occupation \\
\hline Janvier & 101 & 76 & 76 \\
\hline Février & 92 & 83 & 90 \\
\hline Mars & 101 & 91 & 89 \\
\hline Avril & 98 & 91 & 92 \\
\hline Mai & 102 & 110 & 108 \\
\hline Juin & 99 & 112 & 113 \\
\hline Juillet & 102 & 112 & 109 \\
\hline Aoùt & 102 & 130 & 127 \\
\hline Septembre & 99 & 124 & 124 \\
\hline Octobre & 102 & 115 & 112 \\
\hline Novembre & 98 & 92 & 94 \\
\hline Decembre & 101 & 65 & 64 \\
\hline
\end{tabular}

* Coefficients saisonniers calculés à l'aide de la procédure X-1 I du logiciel SAS.

TABLEAU 5

Prévisions mensuelles des chambres disponibles à Montréal de janvier 1988 à mars 1990

\begin{tabular}{|c|c|c|c|c|}
\hline Annés & Mois disponibles & $\begin{array}{c}\text { Chambres } \\
\text { prévues }\end{array}$ & Chambres & Ecarts \\
\hline \multirow[t]{12}{*}{1988} & JAN & 392429 & 390467 & 1962 \\
\hline & FÉV & 366096 & 35,3108 & 12988 \\
\hline & MARS & 390755 & 391709 & $=954$ \\
\hline & AVRIL. & 379050 & 378535 & 515 \\
\hline & MAI & 393049 & 387900 & 5149 \\
\hline & JUIN & 380580 & 378426 & 2154 \\
\hline & JUIL. & 393297 & 392842 & 455 \\
\hline & AOUT & 393297 & 394916 & -1618 \\
\hline & SEPT & 380610 & 382334 & -1724 \\
\hline & OCT & 387097 & 389613 & .2516 \\
\hline & NoV & 373890 & 372417 & 1473 \\
\hline & DEC & 382974 & 381876 & 1098 \\
\hline \multirow[t]{12}{*}{1989} & JAN & 391437 & 387649 & 3788 \\
\hline & FÉV & 354820 & 351914 & 2906 \\
\hline & MARS & 380189 & 385296 & -5106 \\
\hline & AVRIL. & 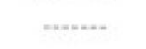 & 370783 & $\ldots$ \\
\hline & MAl & …… & 381,334 & ...... \\
\hline & JUIN & & 371028 & $\ldots$ \\
\hline & JUIL & & 384983 & $\ldots$ \\
\hline & AOUT & & 388656 & \\
\hline & SEPT & -...- & 378772 & -.... \\
\hline & OCT & .....- & 388327 & $\ldots$ \\
\hline & NOV & .....-- & 373614 & 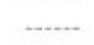 \\
\hline & DÉC & -...... & 382851 & ..... \\
\hline \multirow[t]{3}{*}{1990} & IAN & ....... & 388845 & -..- \\
\hline & FÉV & - & 351627 & $\ldots-$ \\
\hline & MARS & $-\ldots$ & 383268 & $-\ldots$ \\
\hline
\end{tabular}

même temps que l'estimation des coefficients à travers une procédure de régression de type Stepwise (pas à pas).

La méthode XII a été développéc surtout par Julius Siskin du Bureau de recensement des Etats-Unis dans les années 1960 . Elle consiste par les moyennes mobiles (et autres techniques statistiques), à extraire les differents mouvements des séries chronologiques et àl leur donner une forme normalisée. On peut par la suite intégrer ces mouvements à nos projections".

La méthode X11 foumit aussi des prévisions pour la composante saisonnière. Avant d'être réassociée à la composante saisonnière pour effectucr les prévisions, la composante désaisonnaliscé issuc de $\mathrm{X} 11$ a été traitée sous forme de modèle tendanciel estimé par régression (méthode 4) ou sous forme de modèle tendanciel et autorégressif estimé par la méthode Stepar (methode 5).

Dans les trois séries traitées dans ce document. la composante saisonnière et la composante désaisonnalisée sont associées sous forme multiplicative. On peut résumer l'ensemble de ces approches par le tableau 2.

Dans celte étude, nous avons donc expérimenté 15 ajustements possibles de la série temporelle. Pour chacune des méthodes, nous avons eu recours aux hypothèses de stabilité, d'évolution linéaire et d'évolution parabolique de la tendance.

Les 2 dernières méthodes ( 4 et 5 ) comportent chacune trois tapes:

la dissociation du facteur saisonnier et de la série désáisonnal isée;

- le traitement de la série désaisonnalisée par la régression (méthode 4) ou par Stepar (méthode 5). La régularité de la série désaisonnalisée a elle aussi été étudiée à partir des hypothèses de stabilité, d'évolution linéaire et d'évolution parabolique:

- la réassociation prévisionnelle du facteur saisonnier et de la série désaisonnalisée.

\section{Évolution récente}

Les chambres d'hôtels disponibles à Montréal ont augmenté lentement entre 1977 et 1988 (voir le tableau 3); le taux d'accroissement annuel moyen" a été de $2 \%$. Deux périodes de baisse ont rythmé cette évolution: elles se situent en 1981 et en 1984-1985. Depuis 1985, il y at une progression continue des chambres disponibles d'environ $5 \%$ par an en moyenne.

Les nuitées ont connu une longue baisse en 1981-1982-1983. Depuis 1984, lil reprise est relativement forte. Les taux d'occupation suivent la même progression et se maintiennent malgré l'augmentation constante des chambres dis= ponibles. L'augmentation graduelle des nuitées 
a été assez bien anticipée par les investisseurs: dans ce sens, le taux d'occupation est un bon indicateur de l'évolution du marché dans l'industrie hôtelière montréalaise.

\section{Étude de la saisonnalité}

Dans l'ensemble, les chambres disponibles subissent peu de variations liées à des phénomènes saisonniers (voir tableau 4). Lat valeur la plus faible est en février, les valeurs les plus hautes sont nombreuses et varient entre 100 et 102 (ce qui est très léger).

Les oscillations dues aux saisons sont plus fortes pour les nuitées; la valeur la plus faible se situe en décembre et la valeur la plus élevée en août (l'ecart entre la valeur la plus haute et la valeur la plus faible est de 65 points). Les mois de mars, avril et novembre sont assez près des moyennes annuelles. Les hôteliers disposent d'au moins six mois de relative prospérité (de mai à octobre) avèc des mois de restrictions en décembre, janvier et février.

A de légères variantes près, les coefficients saisonniers des taux doccupation sont la fidèle réplique des coefficients saisonniers des nuitées. On remarque, dans l'ensemble, que la période touristique tend à s'étendre aux extrếmitếs: en mati et en octobre ce qui est different de la période touristique dans le reste du Québec; celle-ci est souvent limitée à la période juinaัoût.

\section{Étude des prévisions}

Dans le tableau 5, nous présentons les prévisions mensuelles des chambres disponibles pour l'année 1989 et les trois premiers mois de 1990. Les meilleures prévisions proviennent de l'approche $\mathrm{X}-11$ associée à Stepar, utilisant l'hypothèse d'une tendance linéaire (ceci est aussi valable pour les prévisions des nuitées et des taux d'occupation). Toute chose étant égale par ailleurs, il faut s'attendre à une très légère baisse des chambres disponibles de l'ordre d'environ $1.5 \%$ pour l'ensemble de l'année 1989.

Le tableau 6 montre les prévisions mensuelles des nuitées en 1989 et une partie de 1990. Le modèle suggère l'hypothèse qu'il faut s'attendre à une certaine baisse conjoncturelle des nuitées en mars et aoút 1989. Il y aura ensuite une faible reprise pour les demiers mois de 1989 et les premiers mois de 1990.

On peut voir dans le tableau 7 les prévisions mensuelles des taux doccupation; ceux-ci sont calculés de façon autonome par rapport à la série des chambres disponibles et à la série des nuitées. Dans le tableau 8 , nous avons les prévisions mensuelles des taux d'occupation calculés cette fois à partir des prévisions des chambres disponibles et des prévisions des nuitées. Les différences sont subtiles entre les deux formes de prévision; elles découlent nettement des

TABLEAU 6

Prévisions mensuelles des nuitées à Montréal de janvier 1988 à mars 1990

\begin{tabular}{|c|c|c|c|c|}
\hline Années & Mois & $\begin{array}{l}\text { Chambres } \\
\text { disponibles }\end{array}$ & $\begin{array}{c}\text { Chambres } \\
\text { prévues }\end{array}$ & Ecarts \\
\hline \multirow[t]{12}{*}{1988} & $J A N$ & 198388 & 194345 & 4043 \\
\hline & FEV & 213908 & 215664 & -1756 \\
\hline & MARS & 258233 & 242722 & 15511 \\
\hline & AVRIL. & 245431 & 241926 & 3505 \\
\hline & MAI & 295715 & 298950 & -3235 \\
\hline & JUIN & 292906 & 295754 & -2848 \\
\hline & JUIL & 293475 & 297556 & -4081 \\
\hline & AOUT & 340408 & 341929 & -1.521 \\
\hline & SEPT & 318251 & 326386 & -8135 \\
\hline & OCT & 289913 & 296986 & -7073 \\
\hline & NOV & $232 \quad 184$ & 236534 & -4350 \\
\hline & DEC & 193254 & 169597 & 23657 \\
\hline \multirow[t]{12}{*}{1989} & JAN & 198784 & 203115 & -4331 \\
\hline & FEV & 224008 & 222.590 & 1418 \\
\hline & MARS & 214384 & 246915 & -32531 \\
\hline & AVRIL & -....... & 238079 & -....- \\
\hline & MAI & ........ & 284522 & $\ldots$ \\
\hline & JUIN & $\ldots$ & 289922 & $\ldots=$ \\
\hline & JUIL. & …… & 284096 & $\ldots . .$. \\
\hline & AOUT & …… & 336704 & $\ldots$ \\
\hline & SEPT & - & 328608 & $\ldots$ \\
\hline & OCT & -...... & 299273 & мими \\
\hline & NOV & -..... & 241677 & n..... \\
\hline & DÉC & -n. & 168057 & 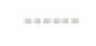 \\
\hline \multirow[t]{3}{*}{1990} & JAN & ....... & 196854 & ........ \\
\hline & FÉV & -..... & 214260 & $\ldots$ \\
\hline & MARS & $\ldots$ & 244179 & ...... \\
\hline
\end{tabular}

procédures utilisćes. Néanmoins, les prévisions effectućes à partir de la série brute des taux cumulent de façon aveugle les fluctuations des chambres disponibles et des nuitées.

Les prévisions des taux calculés à partir des prévisions effectuées par les nuitées et sur les chambres disponibles sont en principe plus fiables parce que les deux séries comportent des informations plus homogènes et que chaque série représente un volet (offre ou demande) distinct du marchê.

\section{Évolution du marché hôtelier à Montréal}

La construction de nouveaux hôtels dans les trois dernières années a modifié la physionomie du marché hôtelierà Montréal. Par anticipation, l"industrie hơtclière s'est ajustée à la demande de chambres en croissance. Ces ajustements successifs apparaissent clairement dans le tableau 9 où nous avons défini certaines hypothèses de travail.
Dans Thypothese I (colonne 3 du tableau 9). nous avons conservé les chambres disponibles en 1986 et dans l'hypothèse 2 (colonne 4 du tableau 9), les chambres disponibles en 1987. En fonction de ces deux hypothèses, nous avons calculé les taux d'occupation à partir des nuitées prévues pour 1989 et 1990 . Lacolonne 5 présente les taux résultant de l'hypothèse 1 et la colonne 6 les taux calculés en tenant compte de la deuxième hypothèse.

Dans l'hypothèse 1 , on fait comme s'il n'y avait cu aucune construction, ni destruction ou modification d'hôtelsen 1986. Dans l'hypothèse 2, on se donne un préjugé semblable mais à partir de l'année 1987.

On remarque que si on maintient le pare des chambres disponibles au même niveau qu'en 1986, Ic taux d'occupation, par exemple en avril 1989 , serait de 70.5 ; de la mème façon, si on conserve le même nombre de chambres disponibles, on obtient pour avril 1989 le taux de 
TABLEAU 7

Prévisions mensuelles des taux d'occupation des hôtels de Montreal de janvier 1988 à mars 1990

\begin{tabular}{|c|c|c|c|c|}
\hline Années & Monts & $\begin{array}{l}\text { Chambres } \\
\text { disponibles }\end{array}$ & $\begin{array}{l}\text { Chambirs } \\
\text { preveres }\end{array}$ & Eeart \\
\hline \multirow{12}{*}{ 10AS } & JAN & (8) & 5 & -1 \\
\hline & $\mathrm{Fev}$ & 58 & 59 & -1 \\
\hline & MARS & $\alpha$ & 60 & 6 \\
\hline & AVkil. & 65 & की & -2 \\
\hline & MAI & 73 & 76 & -1 \\
\hline & JUIN & $n$ & 76 & 1 \\
\hline & JuL. & 75 & 76 & -1 \\
\hline & AOUT & 86 & 86 & 0 \\
\hline & SEPT & 84 & 83 & 1 \\
\hline & OCT & 75 & 76. & -1 \\
\hline & Nov & 62 & 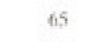 & -3 \\
\hline & DEC & 81 & 43 & 2 \\
\hline \multirow[t]{12}{*}{1989} & IMN & 60 & 55 & 5 \\
\hline & FEY & $\alpha s$ & is & 3 \\
\hline & MARSS & 54 & 62 & -8 \\
\hline & AVkIL & & 63 & $\cdots$ \\
\hline & Mal & - & 76 & -- \\
\hline & IUIN & & 77 & $=$ \\
\hline & лиน. & & $n$ & \\
\hline & AOUT & & 42 & \\
\hline & SEIYT & & 88 & \\
\hline & OCT & & 76 & - \\
\hline & Nor & & th & \\
\hline & & & & \\
\hline \multirow[t]{2}{*}{ 1940, } & JAN & & 51 & - \\
\hline & $\begin{array}{l}\text { FEV } \\
\text { MAKS }\end{array}$ & $=$ & 59 & $-=$ \\
\hline
\end{tabular}

\section{TABLEAU 8}

Taux prévus pour les hôtels de Montréal calculés à partir des chambres disponibles prévues (tableau 5) et des nuitées prévues (tableau 6)

\begin{tabular}{|c|c|c|}
\hline Annees & Mois & Taux prevus \\
\hline \multirow[t]{9}{*}{ 198\% } & AVRIL. & 6042 \\
\hline & MAI & 746 \\
\hline & JUIN & 78.1 \\
\hline & MULLET & 73.8 \\
\hline & ROUT & 866.6 \\
\hline & SEPTEMAREE & 86.8 \\
\hline & OCTOBRE & 77.1 \\
\hline & MONEMBRE & $\$ 4.7$ \\
\hline & DÉCEMIREE & 43.9 \\
\hline \multirow[t]{3}{*}{1990} & IANWIER & 50.6 \\
\hline & FEVRIER & 60.9 \\
\hline & MARS & 63.7 \\
\hline
\end{tabular}

66.2. On peut aussi comparer les taux issus de ces deux hypotheses aux taux prévus (colonne 7 ).

On peut ainsi constater qu'ì chaque fois que les nuitées augmentent, l'industrie hôtelière śajuste en adaptant son stock de chambres disponibles de façon a réduire les frictions sur le marché. II s'agit d'un véritable processus d'anticipation qui indique en mème temps le niveau général de rentabilité. Face à ces données, on ne peut éviter de se poser la question: quel csi le taux d'occupation acceptable (ou plancher) ou nécessaire pour assurer la vitalité dé l'industrie hôtelière?
TABLEAU 9

Hypothèses sur les taux prévus d'occupation des hôtels de Montréal

\begin{tabular}{|c|c|c|c|c|c|c|}
\hline i & 2 & 3 & 4 & 5 & 6 & 7 \\
\hline anones & 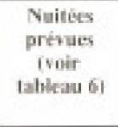 & $\begin{array}{l}\text { Hypothise } \\
\text { 1; chanhles } \\
\text { dispounibles } \\
\text { et 1986 }\end{array}$ & $\begin{array}{l}\text { Hypothese } \\
\text { 2. chambires } \\
\text { disponihles } \\
\text { en } 1987\end{array}$ & $\begin{array}{l}\text { Hyputhès } \\
\text { I: taus con } \\
1580 \text { at } 1990\end{array}$ & $\begin{array}{l}\text { Hypothise } \\
\text { 2: taux es } \\
1989 \text { et } 1990\end{array}$ & $\begin{array}{l}\text { Taux prévu } \\
\text { (voir } \\
\text { tableau sy }\end{array}$ \\
\hline AVR S9 & 238079 & 337650 & 354760 & 76.5 & 66,2 & 64.2 \\
\hline MAI 84 & 284522 & 348915 & 318931 & 81.5 & 37.1 & 746 \\
\hline JUIN S4 $^{2}$ & 280922 & 341 43 & 354760 & BA. 4 & 80.6 & T8.1 \\
\hline JUL By & 2840196 & 347448 & 371752 & $\$ 1.8$ & $\pi n .4$ & 73,8 \\
\hline NOLT & 336704 & 359476 & 571752 & 93.7 & 90.6 & seth \\
\hline SEPT 89 & 3286 6nd & 348801 & 379650 & 94.2 & 86.6 & 868 \\
\hline CKT 89 & 299273 & 361243 & 392305 & 82.8 & 76.2 & $\pi 3.1$ \\
\hline NOY 89 & 24167 & 349500 & $37 \times 870$ & $\omega .1$ & 63.8 & 64.7 \\
\hline bete 89 & 168057 & 3,1243 & 188678 & 46.9 & 43.5 & 43.9 \\
\hline IAV QII & 19685 & 351109 & 371132 & 56.1 & 534 & 510.6 \\
\hline FEV 90 & 214260 & 317212 & 315216 & 67.5 & 639 & foll, 5 \\
\hline MAK 40 & 244179 & 345905 & 3711.32 & 701 & 6.5 .8 & 6.3 .7 \\
\hline
\end{tabular}

\section{NOTES EXPLICATIVES}

(1) Nows remercions l'Association ules hótels du Grand Montréal des renseignements mis ì notre disposition.

12) Voür dans: CLUZEAU, P., Le Québec touristique. Indicateurs sur les marchés et les secteurs touriatiques de 1980 a 1988, Les Publications du Québoc, Québec, 1989.

(3) Extrait de: BODSON, P., SAMSON, M., ST MFORD, J L' hòtellerie dans I'arrondis. sement-Centre de Montréal: situation et perspectives d'avenir, Ville de Montréal, Dossies Moxitrúal 6, Montréal, novembre 1989, p, 27.

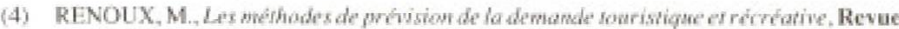
de tourisme, vol, 8, no 1. Beme, 1973.

(5) ARCHER, B, Demand Forecasting in Tourism, Universily of Wales Peas, Bangor, 1976

(6) BAR-ON, V.. Seasunality in Tourism - A Guide to the Analysis of Seasonality and Trends for Poliey Making. The Ecomomis Intelligense Unit Limited, London, 1975; et aussid du mème autcur: Fowecesting Tourism and Thavel Series, Problemy Turystyki. vol. 7. no 3. Wanzasa, 1984.

(7) CHOY, J. Farecasring Tourism Revisidw. Tourism Managenent, Sumey, wol. 5, no 3, 1984

(8) MARTIN, C, WITT, S., An Empirical Analysis of the Accuracy of Forecasting Technique, The Travel and Tsurism Research Association. Ninetecnth Annual Conference, Montreal. June 19-23, 1988.

(4) Nous cmprustons catte distinction al GOURIEROUX. C. MONFORT. A. Cours de séries temporelles. Economica, Paris, 1983, p, 14

(10) Voir: MAKRIDAKIS, S. WHEELWRIGHT. S. Methodes de previcion pour la gestion, Les Editions de l'Organisation, Puris, 1983, pp, 72-89.

(11) Dapres: LEWANDOWSKI, R., La prevision a court terme, Dunod, Paris, 1979, pp. 9293.

(12) Stepar pour Stepwise Autoregressiwe Method.

(13) IEWANDOWSKL, R., op. cit, p. 102 .

(14) MAKRIDAKIS, S, WHEELWRIGHT, S., McGFF, V., Forecasting: Methods and Applications, John Wiley and Sons, N.Y., 1983, pp, 149.177.

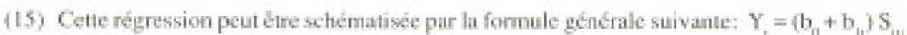
$+\mathrm{E}$

(16) Il sitgit de la moysane géontétrique pour le calcul du taux d'accroissement annuel moyen. 